\title{
Current-Sensor-Based Feed Cutting Force Intelligent Estimation and Tool Wear Condition Monitoring
}

\author{
Xiaoli Li, Alexandar Djordjevich, and Patri K. Venuvinod
}

\begin{abstract}
Tool wear condition monitoring has the potential to play a critical role in ensuring the dimensional accuracy of the workpiece and prevention of damage to cutting equipment. It could also help in automating cutting processes. In this paper, the feed cutting force estimated with the aid of an inexpensive current sensor installed on the ac servomotor of a computerized numerical control turning center is used to monitor tool wear condition. To achieve this, the feed drive system is modeled, using neuro-fuzzy techniques, to provide the framework for estimating the feed cutting force based on the feed motor current measured. Functional dependence of the feed cutting force on tool wear and cutting parameters are then expressed in the form of a difference equation relating variation in the feed cutting force to tool wear rate. The computerized system automatically compares successive feed cutting force estimates and determines the onset of accelerated tool wear in order to issue a request for tool replacement. Experimental results show that the tool wear condition monitoring is effective and industrially applicable.
\end{abstract}

Index Terms-Feed cutting force, monitoring, neuro-fuzzy, tool wear.

\section{INTRODUCTION}

A RELIABLE AND inexpensive tool monitoring system for cutting processes would contribute to the effective utilization of the machine tools, cutting tools, and workpieces. However, current tool monitoring solutions have unacceptably high cost/performance ratios and are effective only within a limited range of cutting conditions [1], [8]. Moreover, cutting force measurement using dynamometers, which has emerged as one of the most popular means of monitoring tool wear, is difficult to apply in a production environment because of its high cost, negative impact on cutting system rigidity, the requirement for a wiring harness, and the limitation it imposes on machining stroke lengths [2]. In order to overcome these disadvantages, we propose a system based on motor current signals and an adaptive neuro-fuzzy inference system (ANFIS) for online estimation of cutting forces. These force estimates, in turn, allow the evaluation of the tool wear rate and, consequently, enable tool condition monitoring.

Current sensing by itself is not new in metal cutting. Stein et al. [3] have developed a dynamic model with lumped parameters that capture the essential characteristics of the dc servo

Manuscript received July 27, 1999; revised December 12, 1999. Abstract published on the Internet March 12, 2000.

$\mathrm{X}$. Li is with the Institute of Precision Engineering, Harbin Institute of Technology, Harbin 150001, China.

A. Djordjevich and and P. K. Venuvinod are with the Department of Manufacturing Engineering and Engineering Management, City University of Hong Kong, Hong Kong.

Publisher Item Identifier S 0278-0046(00)04756-0. feed drive system and have studied the bandwidth, sensitivity, and accuracy associated with the process of measuring the cutting force in turning by monitoring the respective motor current. Altintas [4], [5] has analyzed a feed drive control system of machine tools in detail and the feasibility of using armature current as a cutting force sensor signal. Lee et al. [2], [6] have introduced real-time tool-breakage detection for numerical control (NC) milling by monitoring the cutting force that is derived from the current signal of the feed drive ac motor. Modeling of the feed drive system and model calibration with a tool dynamometer showed that the sensitivity of the feed drive motor current was sufficient to characterize tool breakage. Chang [7] has proposed a neural network to construct a model of the spindle and estimate the cutting force based on the current of the spindle motor. Only a minor alteration of the machine is required while installing the current sensor. In this approach, the feed cutting force is estimated from the feed motor current using neuro-fuzzy techniques. While this work has generally been successful in monitoring the cutting feed force and predicting tool breakage, we now move a small step further and detect the onset of the stage of the tool's poor performance that precedes its breakage, i.e., the accelerated tool wear, before it has severely affected workpiece accuracy.

A review of the literature on cutting reveals that the cutting force is mainly a function of the cutting speed $v$, feed rate $f$, depth of cut $d$, and an appropriate tool wear measure $w$. In turn, the tool wear rate $\dot{w}$ depends on these same cutting parameters $(v, f, d)$. Equally importantly, there usually is a direct influence of tool wear on the magnitudes of cutting force components, although the nature and magnitude of this influence vary depending on the prevailing mix of wear modes (flank wear, crater wear, edge chipping, tool fracture, groove wear, etc.). For instance, when flank wear occurs, there is additional rubbing action at the flank wear land area so that cutting force increases with increasing wear land length during steady-state wear conditions. Eventually, when the tool reaches the stage of catastrophic wear (the stage of significantly accelerated wear), either the workpiece or the tool softens and, therefore, one can expect a noticeable change in the force trend. Hence, in principle, it is possible to monitor tool wear by measuring the feed cutting force. This paper describes a method of estimating the feed cutting force using a current sensor and a dedicated ANFIS and then calculating the tool wear rate from a well-recognized mathematical model. Based on the knowledge of the feed force, we evaluate whether the tool wear has accelerated and, thus, whether the tool has reached the final stages of its useful life. Our approach has been developed in the context of computerized numerical control (CNC) turning. 


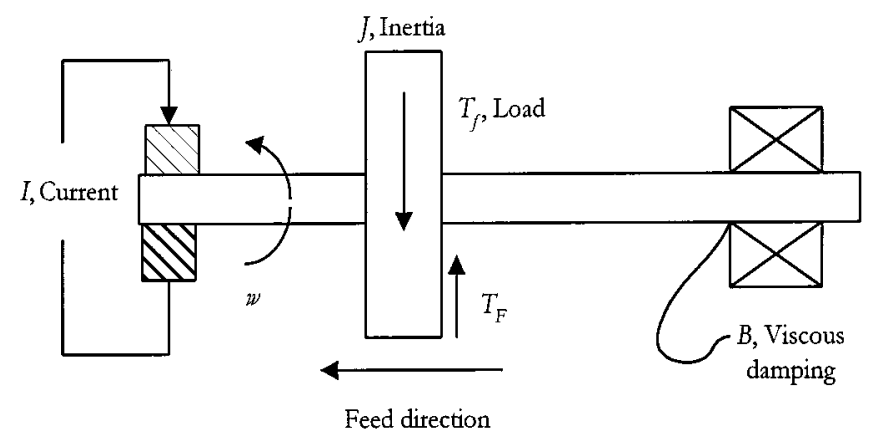

Fig. 1. A simple model of the typical feed drive system.

\section{Estimation OF THE FeEd CutTing ForCE}

\section{A. Modeling of the Feed Drive System}

The typical ac servo feed system on the CNC lathe is composed of the following basic components: cutting tool, tool post and carriage, ball screw, the bearings supporting the ball screw, feed box, feed motor, and lubrication system. A simple model of the feed drive system consisting of these mechanical components is shown in Fig. 1. In the model, the inertias of all moving parts is lumped into an equivalent inertia $J$. Likewise, the overall viscous damping in the absence of the cutting load is lumped into an equivalent viscous damping with damping coefficient $B$. The additional viscous damping caused by the cutting forces and the total dry or coulomb friction are accounted for by a friction torque $T_{F}$. Finally, the feed force component is represented as a generalized disturbance torque on the feed motor $T_{f}$.

In steady state, the equation of motion of the feed drive system can be expressed as

$$
K I=B \omega+T_{F}+T_{f}
$$

where $\omega$ is the angular velocity and $K$ is the feed motor constant.

The friction torque $T_{F}$ can be expressed as

$$
T_{F}=T_{F 0}+\Delta T_{F}+\Delta T_{V}
$$

where $T_{F 0}$ is the coulomb friction torque in the air-cutting condition and $\Delta T_{F}$ and $\Delta T_{V}$ are the additional coulomb and viscous friction torques due to the cutting load, respectively.

The feed force $F_{f}$, and its corresponding torque $T_{f}$ in (1) are functionally related

$$
T_{f}=\frac{p}{2 \pi \mu} F_{f}
$$

where $\mu$ and $p$ are the mechanical efficiency and pitch (length/rotation) of the ball screw, respectively.

Substituting (3) into (1), it becomes apparent that the feed force $F_{f}$ depends on the motor drive current $I$.

\section{B. Estimation of the Air-Cutting Current}

Force $F_{f}$ (or torque $T_{f}$ ) in (1) will be estimated based on the corresponding "cutting current" $\Delta I=I-I_{0}$ where $I$ is the magnitude of the measured total current and $I_{0}$ is the "aircutting current" that is consumed when there is no cutting load.

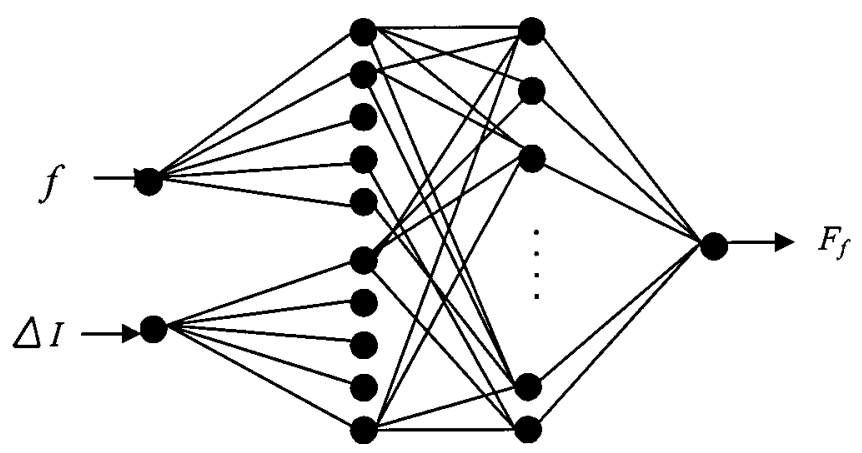

Fig. 2. Structure of the ANFIS used for the estimation of the feed cutting force $F_{f}$.

Experiments have shown that the air-cutting current $I_{0}$ varies linearly with the feed speed, $f$ (millimeters per minute)

$$
I_{0}=-0.0007 f+0.3476 \text {. }
$$

The magnitudes of the coefficients in this linear equation have been obtained by performing a linear regression exercise over the measured data points.

In the case of air cutting, following (1), we have $K I_{0}=B \omega+$ $T_{F 0}$. By subtracting this expression from (1), it follows that

$$
K \Delta I=T_{f}+\Delta T_{F}+\Delta T_{v} .
$$

\section{Feed Cutting Force versus Cutting Current and Feed Speed}

Because the air-cutting current $I_{0}$ has been estimated by reference to (4), the cutting current $\Delta I=I-I_{0}$ can be calculated from the measured value of the total current $I$. On the other hand, since the additional friction torques $\Delta T_{F}$ and $\Delta T_{v}$ depend on the feed cutting force and feed speed, the feed force $F_{f}$ can be expressed as a function (initially unknown) of $\Delta I$ and $f$

$$
F_{f}=F(\Delta I, f) .
$$

Equation (6) indicates that it is important to recognize that the feed cutting force depends on the cutting current and feed speed and this dependence cannot be anticipated from explicit modeling alone.

The function $F(\Delta I, f)$ in (6) may be learned through machine training (see Section II-D). In doing so, we may also extract feed rate $f$ from the current signal so as to enable a more convenient data input to the monitoring system. As expected, experiments have confirmed that the frequency of the cutting current signal varies linearly with the feed speed

$$
f=0.3586 s
$$

where $s$ is the frequency of the ac feed-motor current signal.

\section{Estimation of the Cutting Force $F_{f}$}

We used an ANFIS to map the functional dependence of the feed cutting force $F_{f}$ on the cutting current $\Delta I$ and feed speed $f$ [expression (6)]. The structure of the ANFIS is shown in Fig. 2.

With our ANFIS in place, the feed cutting force can be estimated by reference to the overall monitoring system model 


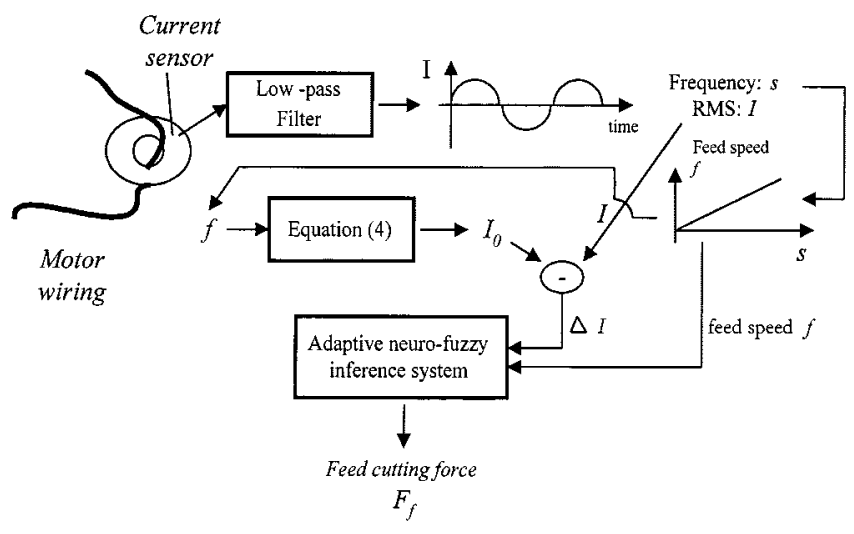

Fig. 3. The overall scheme of the system for estimating the feed cutting force $F_{f}$.

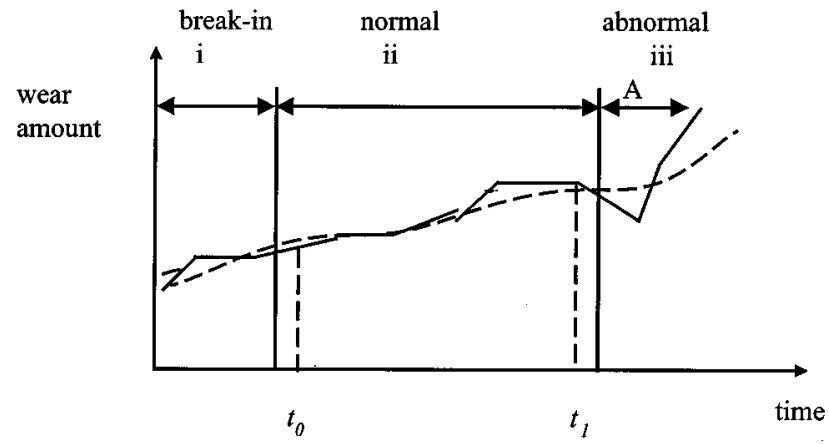

Fig. 4. Tool wear phases.

frame shown in Fig. 3. The feed-motor current was measured by a printed-circuit-board (PCB)-mounted Hall-effect current transducer. Following low-pass filtering, frequency $s$ and rms of the current signal $I$ are obtained.

The feed speed $f$ [see (7)] and the air-cutting current [see (4)] were calculated. After due training, the ANFIS was able to output an estimate of the feed cutting force $F_{f}$ each time a pair of $f$ and $I_{0}$ values were input. In our work, five fuzzy partitions, all triangular in shape, for the two input variables $(\Delta I$ and $f$ ) were used. ANFIS training completed at epoch 1000, with the minimal training root-mean-square error (RMSE) of 0.01 . Seventy sets of experimental data were collected.

\section{TOOL WEAR MONITORING}

\section{A. Modeling}

It has been recognized widely that the tool life can be divided into three phases characterized by three different wear processes: $i$ ) break-in; ii) normal wear; and iii) abnormal or catastrophic wear (see Fig. 4). The sudden rise in the wear rate observed during the abnormal tool wear phase (phase ii) is of interest here as an indication of the need for tool replacement. Because many factors affect tool wear, the wear curve usually fluctuates and is not smooth.

For practical tool monitoring, the wear amount during normal wear phase $w$ is expressed as a sum of two parts. The first part is the determinable linear increase $\dot{w} t$ that is superimposed onto the second, stochastic part $w_{0}$

$$
w=w_{0}+\dot{w} t
$$

where $w$ (millimeters) is the tool wear value, $w_{0}$ (millimeters) is the initial wear, $\dot{w}$ (millimeters per minute) is the wear rate in the second phase (millimeters per minute), and $t$ (minutes) is the cutting time.

As noted already, the present objective is to monitor the rise in the wear rate associated with the final stage of the accelerated tool wear in phase iii (see Fig. 4) so that a tool replacement decision could be made. We propose that this be achieved indirectly by monitoring the feed cutting current $I$ that is related to the tool wear $w$ through the feed cutting force $F_{f}$. Note that it is not necessary to know the two functional dependencies required exactly, since only the onset of the sudden rise in the rate of change (acceleration) is intended to be detected. Hence, the dependence of the feed cutting force on tool wear can be modeled in this specific context as a linear function

$$
F_{f}=F_{0}+K w
$$

where $F_{0}$ is the feed cutting force arising under identical cutting conditions but with an unworn cutting tool (i.e., when $w=0$ ) and $K$ is a parameter dependent on the cutting speed $v$, feed rate $f$, and depth of cut $d$.

Combining (8) and (9), we obtain

$$
F_{f}=F_{0}+K w_{0}+K \dot{w} t .
$$

Differentiating the terms in (10), we can express a change in the feed cutting force $\Delta F_{f}$ as

$$
\Delta F_{f}=K \dot{w} \Delta t .
$$

Rearranging (11a)

$$
\dot{w}=\frac{\Delta F_{f}}{K \Delta t} .
$$

It follows from (11b) that the detection of a noticeable change in thus calculated wear rate $\dot{w}$ should, in principle, indicate that the tool is in its final stage of the accelerated wear (phase iii of Fig. 4) and should be replaced. In reality, however, the wear rate thus calculated fluctuates, since $F_{f}$ is noisy. This complicates the evaluation of the normal value of the wear rate that needs to be used as a reference while establishing the onset of its further abnormal increase.

\section{B. Strategy of Monitoring the Tool Wear}

In order to evaluate the normal tool wear rate based on (11b) in the presence of significant noise in the feed force signal $F_{f}$, a sufficiently long time interval $\Delta t=N \tau$ is required, where $N$ is an adjustable integer multiplier and $\tau$ is the fixed time base (program scan time) used in the calculations. We select the multiplier $N$ based on prior experience such that this time interval $\Delta t=N \tau$ would be stretched to correspond to the shortest expected normal tool wear phase. The actual monitoring starts with the "normal" wear rate thus established as the reference. 


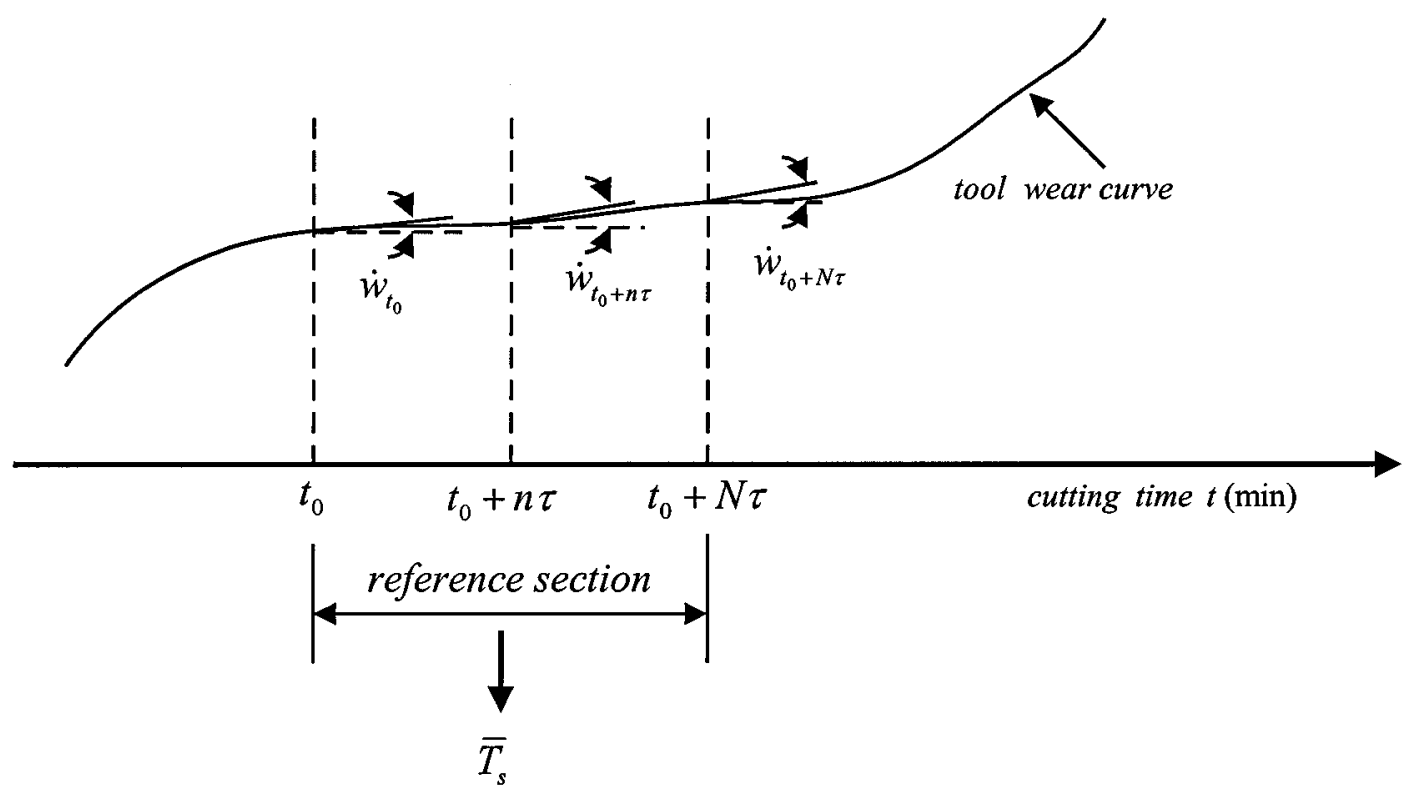

Fig. 5. Schematic diagram of the monitoring strategy.

Each newly calculated wear rate is compared to such a reference regarded as the threshold; a repeatedly high difference between the two indicates an abnormal tool wear and the need for changing the tool.

It is difficult to determine the threshold value implicit in the measurements. For this reason, a trend factor $T_{s}(n \tau)$ for the tool wear rate $\dot{w}$ at some instance in time $t_{0}$ is defined as

$$
T_{s}(n \tau)=\dot{w}_{t_{0}+n \tau}-\dot{w}_{t_{0}}
$$

where $n$ is an integer $1 \leq n \leq N$. A form of an average of such trends over all the possible values of $n$ can be defined as

$$
\bar{T}_{s}=\frac{\sum_{n=1}^{N} T_{s}(n \tau)}{N} .
$$

The detailed process is shown in Fig. 5. Finally, when the tool is in its normal wear state, the reference threshold is expressed as

$$
T_{T}=C \bar{T}_{s}+\dot{w}\left(t_{0}\right)
$$

where $C$ is a constant determined by the experiments. Once this threshold has been determined, it is used in monitoring the tool wear condition; when the excess of the tool wear rate $\dot{w}$ over this threshold value $T_{T}$ is registered repeatedly, the tool is judged to have entered its abnormal wear state and has to be replaced.

\section{EXPERIMENTS AND DISCUSSION}

\section{A. Experimental Setup}

Fig. 6 shows a schematic diagram of the experimental setup used. Tests on a $\mathrm{CNC}$ lathe were performed over a range of cutting conditions. The ac feed drive current was measured using a

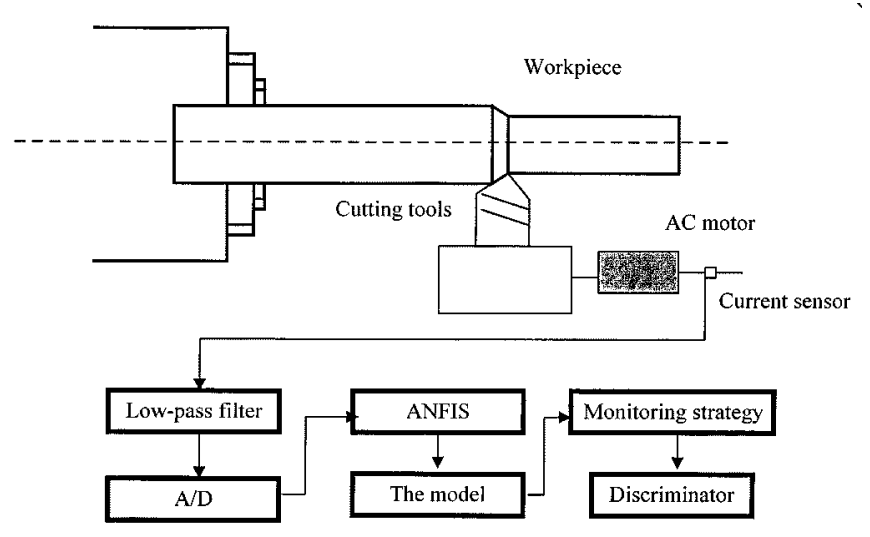

Fig. 6. Schematics of the experimental setup.

Hall-effect sensor. Signals obtained were low-pass filtered (with the cutoff frequency of $100 \mathrm{~Hz}$ ) and A/D converted for processing by a personal computer (Fig. 6).

In order to evaluate the performance of the proposed method, the tests were conducted under the different cutting conditions shown in Table I. The feed cutting force was estimated (based on the current signal and knowledge of the input parameters as described in Section II-D) and its change with time was used for monitoring the tool wear condition.

The record of the actual tool wear with cutting time is shown in Fig. 7. After some 24 min of cutting, when the tool wear amounted to $0.59 \mathrm{~mm}$, the onset of the accelerated wear phase iii (abnormal wear discussed in Section III-A) can be noticed. Our system detected such event by evaluating the wear rate and by comparing it to its precalculated threshold value as illustrated in Fig. 8. According to expectations, by comparison of Figs. 7 and 8 , one can notice that the tool wear rate increases quickly as soon as the tool enters its phase of abnormal wear. In this example, the 
TABLE I

RANGe of CutTing Conditions Used IN TESTS

\begin{tabular}{|c|c|c|}
\hline Tool & Tool material & high-speed steel \\
\hline Cutting & Spindle speed & $1000 \sim 2000 \mathrm{rpm}$ \\
\hline \multirow[t]{3}{*}{ Conditions } & Feed speed & $30 \sim 100 \mathrm{~mm} / \mathrm{min}$ \\
\hline & Depth of cut & $0.5,1,1.5 \mathrm{~mm}$ \\
\hline & \multicolumn{2}{|c|}{ Without coolant } \\
\hline Workpiece & $45 \#$ stec & \\
\hline
\end{tabular}

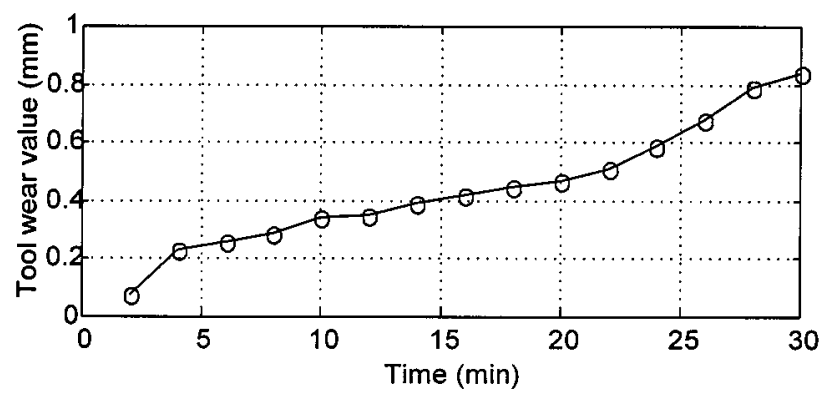

Fig. 7. Observed tool wear with time (workpiece: 45\# steel; spindle speed: $1000 \mathrm{r} / \mathrm{min}$; feed speed: $100 \mathrm{~mm} / \mathrm{min}$; depth of cut: $1 \mathrm{~mm}$ ).

threshold value was calculated at $T_{T}=0.033$. Other parameters were $T_{s}=0.0083$ and $C=2 \mathrm{~mm} / \mathrm{min}$.

\section{B. Discussion}

Although dynamometers can provide accurate measurement of cutting forces and eliminate the need for indirect force estimation, their application in tool monitoring is not practical. Albeit at the cost of more complex computer processing, sensing current of the feed drive represents a much better alternative in terms of the equipment cost, unobtrusiveness, noninterference around the working zone, and retrofitting and hardware simplicity. On the other hand, one limitation of this current-measuring approach is that it cannot be applied for very light cuts because the magnitude of the useful signal is then small and difficult to extract from the total current measured. This total current fluctuates in accordance with the lubrication state and traversing rate of the guideway. However, in such cases, the need for tool monitoring is reduced as well.

The method described does not rely on an exact estimate of the feed force, but on the indication of its relative change (gradient). Hence, the fact that the feed cutting force is estimated with up to $10 \%$ inaccuracy is not detrimental for the tool monitoring in question, in particular, since ANFIS maps the feed force to the corresponding current, with the friction effects accounted for in the model. Moreover, since the wear process is slow in comparison to the time required for execution of the program loop, multiple verifications of the decision to replace the tool can be afforded so as to provide increased confidence in the

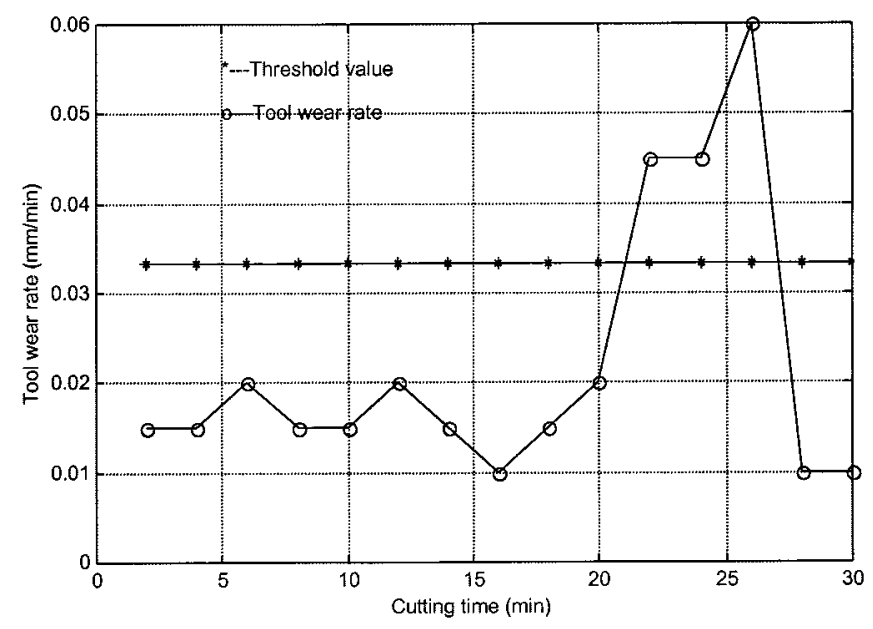

Fig. 8. Observed tool wear rate with time.

decision made (which is equivalent to the averaging of the consecutive force estimates). However, a significant improvement in the accuracy of the force estimate is required if the system were to be used as a dynamometer.

Whatever the cutting conditions and tool and workpiece material, the threshold value for the feed-force gradient is calculated first during the period of known normal tool wear (discussed in Section III-B). Only then is the floating threshold value thus established used in the monitoring process. Such approach allowed the flexibility of self-adaptation across a variety of cutting conditions, work, and tool materials.

\section{CONCLUSIONS}

A tool wear condition monitoring system for turning operations has been proposed that incorporates an adaptive neuro-fuzzy inference. Feed-motor current, measured by a PCB-mounted Hall-effect transducer, is the system input. It is used to estimate the feed cutting force. Functional dependence of this force on tool wear has been analyzed and expressed in a form of a difference equation relating the variation of the feed cutting force to the tool wear rate. By comparing successive feed cutting force estimates, the onset of the accelerated tool wear is determined so that a request for tool replacement could be issued. The method is, in principle, capable of being adaptive to variations in cutting conditions across a wide range of cutting conditions and work and tool materials. The experimental results have shown that this method of tool wear condition monitoring is effective and industrially applicable for all but the very light cuts when the need for such monitoring is reduced. The nature of the hardware used (sensor, in particular) makes the system inexpensive, reliable, and unobtrusive.

\section{REFERENCES}

[1] J. L. Stein and K. Huh, "A design procedure for model-based monitoring systems: Cutting force estimation as a case study," DSC Contr. Manuf. Processes, ASME, vol. 28/PED-52, pp. 45-57, 1991.

[2] J. M. Lee, D. K. Choi, J. Kim, and C. N. Chu, "Real-time tool breakage monitoring for NC milling process," CIRP Ann., vol. 44, no. 1, pp. $59-62,1995$. 
[3] J. L. Stein, D. Colvin, G. Clever, and C.-H. Wang, "Evaluation of dc servo machine tool feed drives as force sensors," Trans. ASME-J. Dynam. Syst., Meas. Contr., vol. 108, no. 4, pp. 279-288, Dec. 1986.

[4] Y. Altintas and C. L. Dong, "Design and analysis of a modular CNC system for machining control and monitoring," in Modeling of Machine Tools: Accuracy, Dynamics, and Control. New York: ASME, 1990, vol. 45, pp. 199-208.

[5] Y. Altintas, "Prediction of cutting forces and tool breakage in milling from feed drive current measurements," Trans. ASME—J. Eng. Ind., vol. 114, no. 4, pp. 386-392, Nov. 1992.

[6] T.-Y. Kim and J. Kim, "Adaptive cutting force control for a machining center by using indirect cutting force measurements," Int. J. Mach. Tools Manuf., vol. 36, no. 8, pp. 925-937, Aug. 1996.

[7] Y.-C. Chang, K.-T. Lee, and H.-Y. Chuang, "Cutting force estimation of spindle motor," J. Contr. Syst. Technol., vol. 3, no. 2, pp. 145-152, June 1995.

[8] D. Li and J. Mathew, "Tool wear and failure monitoring techniques for turning-A review," Int. J. Mach. Tools Manuf., vol. 30, no. 4, pp. $579-598,1990$

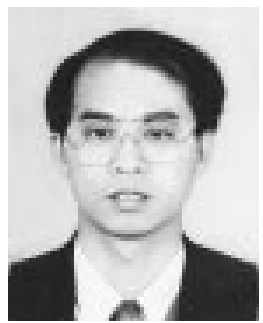

Xiaoli Li was born in Jiangxi, China, in 1970. He received the B.S.E. and M.S.E. degrees from Kunming University of Science and Technology, Kunming, China, and the Ph.D degree from Harbin Institute of Technology, Harbin, China, in 1992, 1995, and 1997, respectively, all in mechanical engineering.

He is currently a Research Fellow in the Department of Manufacturing Engineering and Engineering Management, City University of Hong Kong, Hong Kong. His main areas of research are manufacturing process monitoring, machining error compensation, and fuzzy system, neural network, and wavelet transform and their applications.

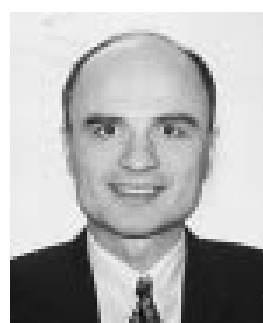

active damping.

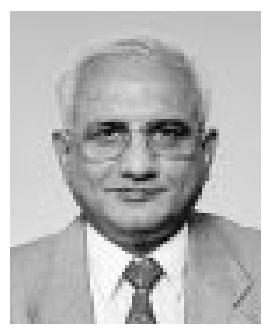

Patri K. Venuvinod has carried out research in the broad field of machining science, machining tools, AI applications in manufacturing, CIM and assembly, supervised numerous Ph.D., M.Phil., and M.Sc. students, and authored more than 90 published scientific papers. He has established research links with a number of overseas and local organizations, and holds an Honorary Professorship at Shandong University, Jinan, China. He has served as a member of numerous government and industrial committees. He has served as Honorary Director of the Institute of Industrial Engineers (Hong Kong) (IIE), Steering Committee Member of the City/HKPC Rapid Prototyping Center, and as a Judge of the HKSAR Award for Productivity. He has organized a number of regional and international conferences, chaired many technical sessions, and served as a reviewer for several international journals and conferences. His current areas of research include compensation of machining errors, fusion of modeling and sensing, intelligent geometric feature recognition, and chip control in machining.

Prof. Venuvinod is a Fellow of the Institution of Electrical Engineers, U.K., and Hong Kong Institution of Engineers. He is a Senior Member of the IIE and an active member of CIRP. 